\title{
Vestibular syndrome after cochlear implantation
}

\author{
Adina ZAMFIR-CHIRU-ANTON ${ }^{1}$, Dan Cristian GHEORGHE2,3 \\ 1"Grigore Alexandrescu" Emergency Hospital for Children, Bucharest, Romania \\ ${ }^{2}$ Children Clinical Emergency Hospital "M. S. Curie", Bucharest, Romania \\ 3University of Medicine and Pharmacy "Carol Davila", Bucharest, Romania
}

\begin{abstract}
Vestibular syndrome after cochlear implantation is described in the literature. Its causes are variate but not always amenable to etiologic approach. We describe a patient which developed cochlear electrode extrusion with persistent vestibular symptoms after a successful insertion.

Material and method. Photos from surgery and CT imaging were used to show this postoperative complication.

Results. Revision surgery was necessary to reinsert and properly immobilize the electrode to the osseous walls of the mastoidectomy cavity. Symptoms disappeared completely after this procedure.

Conclusions. Persistent vestibular symptoms after cochlear surgery require special and continuous monitoring. Revision surgery is indicated wherever imaging investigations suggest possible and correctable causes.
\end{abstract}

Keywords: cochlear implant, vestibular syndrome, revision surgery

\section{INTRODUCTION}

Vestibular syndrome is quite common after cochlear implantation (incidence 2-47\%) (1). It can appear shortly after implantation (in the first 24 hours postoperatively), is mild and regressive (self-limited), and has a short duration (maximum 3 days). Sometimes the onset of vertogo can be delayed and episodic after the surgery (1). The children with vestibular aqueduct larger than $2 \mathrm{~mm}$ in the intraosseous part have a greater risk of developing vestibular syndrome after $\mathrm{Cl}(2)$.

The vestibular syndrome with delayed onset after $\mathrm{Cl}$ can develop as late as 12 months after implantation, can be a consequence of endolymphatic hydrops, can be bilateral and is usually produced by degenerative changes of the inner ear like fibrosis and loss of sensory cells (3).
The risk of developing vestibular syndrome after $\mathrm{Cl}$ depends on the age of the patient (the older the patient is at implantation, the higher the risk of vestibular syndrome postoperatively) (3), on the type and quality of the inserted electrode, on the size of the cochleostomy hole.

The causes of developing vestibular syndrome after $\mathrm{Cl}$ are multiple (4): dispersion of electrical current during activation of the implant, infection of inner ear fluids, developing of a perilymphatic fistula after cochleostomy, loose electrode (floating in and out of the cochleostomy) - as in our case presentation.

\section{CASE REPORT}

A girl aged 15, with bilateral profound sensorineural hearing loss, postlingual type (probably of autoimmune cause) - was investigated by 
BERA, ASSR, CT, MRI and indicated for cochlear impantation in September 2014. The audiologist suggested implantation of the left ear, in order to preserve the residual hearing, which was better in the right ear.

The preoperative imaging showed mild blurring (ossification?) at the top of the left cochlea (Fig. 1, 2).
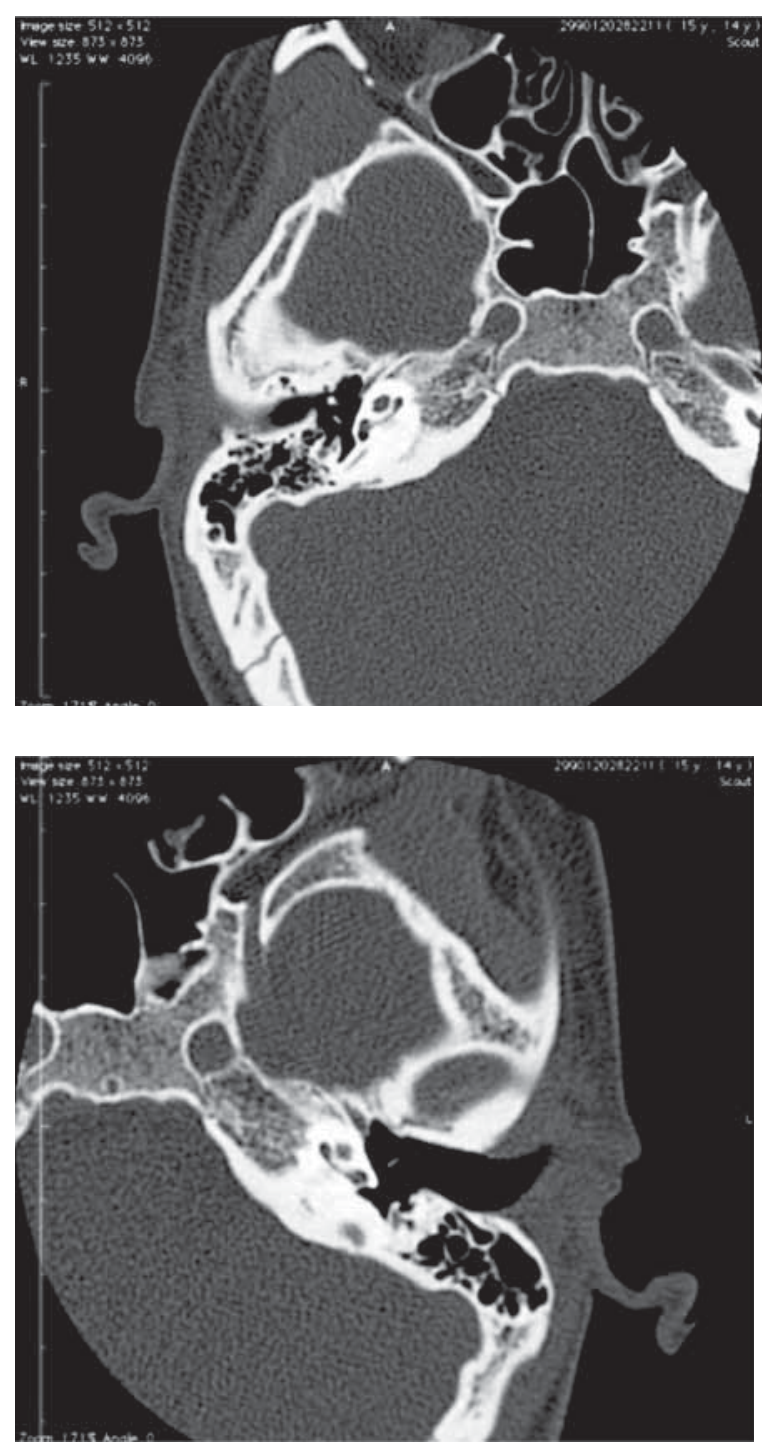

FIGURE 1, 2. Discrete blurring of the cochlear apex on the left side (preoperative CT)

The surgery of the patient performed uneventfully until insertion of the electrode: the MED-EL Sonata FLEX28 electrode could only partially be fitted into the cochleostomy; a Compressed electrode was fully inserted instead (these technical problems made up for about 30 minutes delay, the cochleostomy remained open during this period of time); finally the intraoperative measurements (NRT and tympanometry were normal). The pacient developed postoperatively a severe vestibular syndrome shortly after the operation (had heavy nausea and vomiting, could not stand, could not feed, so parenteral substitution was installed during the first 4 days after surgery). The treatment included iv antibiotics (meronem), antiemetic drugs (granisetron), intravenous fluids. Despite this treatment, the patient's condition got worse.

On the $4^{\text {th }}$ day postoperatively, a control CT scan was performed, showing a intracochlear inserted electrode, but also gas inside the cochlea (suggesting perilymphatic fistula) (Fig. 3, 4).
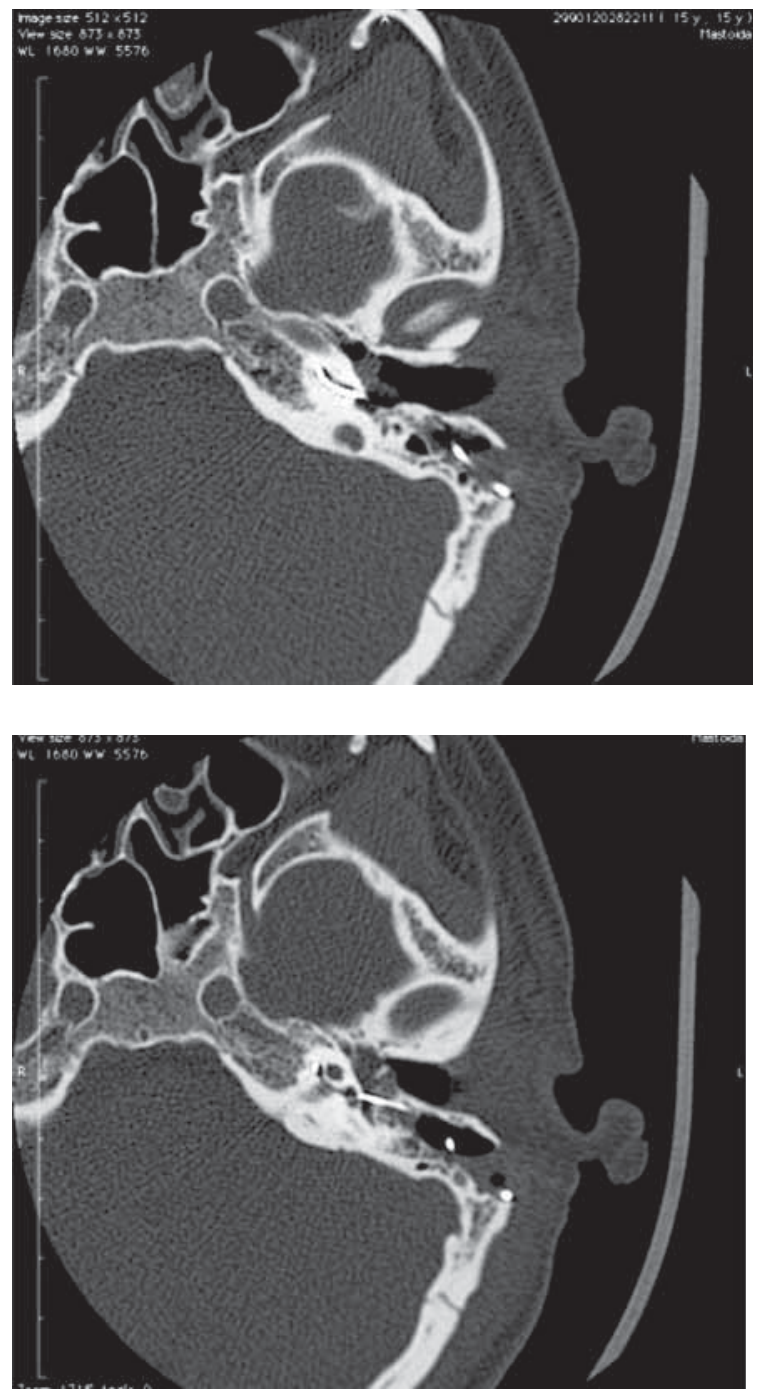

FIGURE 3, 4. Intracochlear electrode array, surrounded by air bubbles scattered on all its length (postoperative CT)

A decision was taken to operate the patient immediately. During revision surgery, we discovered the electrode array partially removed outside of the cochlea, although the sealing fascia was in good position around the cochleostomy (Fig. 5). There were no signs of infection.

The electrode was fully reinserted into the cochleostomy and fixed to the margin of the 


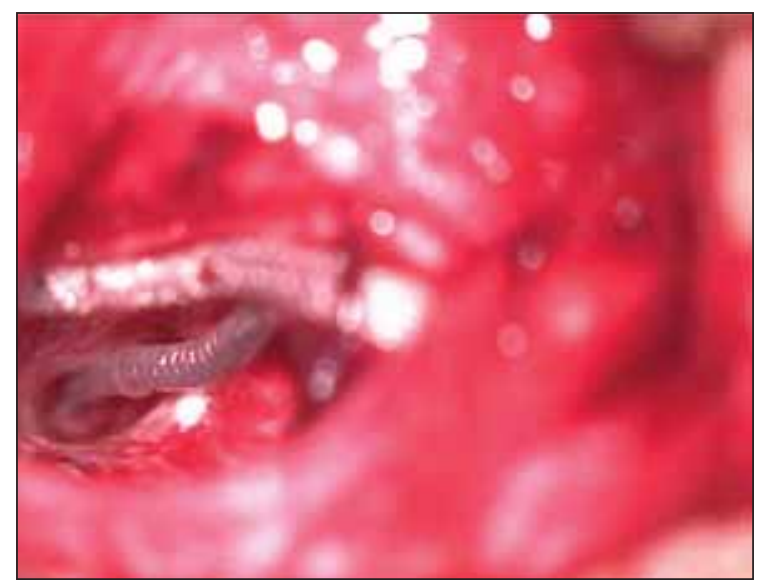

FIGURE 5. Electrode array partially extruded from the cochlea

posterior tympanotomy with KetacCem (glass ionomer cement), after which the cochleostomy was resealed with temporal fascia fragments and surgical (Fig. 6,7).
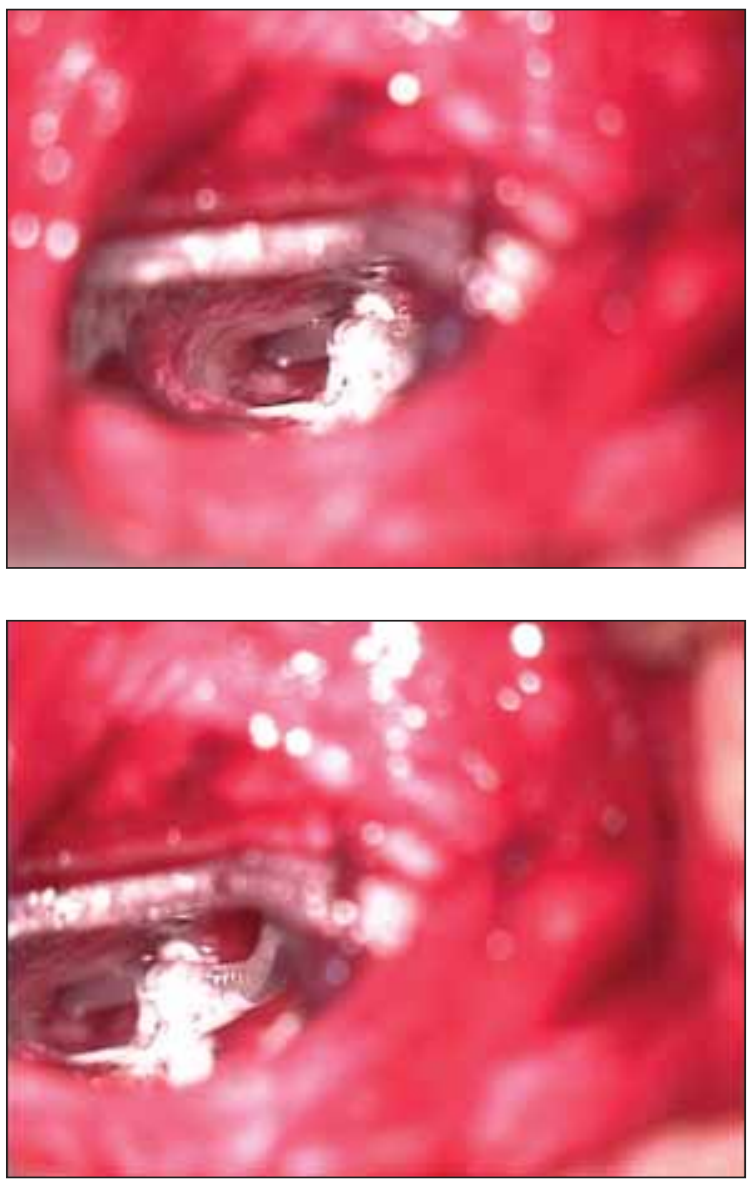

FIGURE 6, 7. Reinsertion of the electrode array and fixing it to the posterior tympanotomy bone with glass ionomer cement
The follow-up after revision surgery was uneventful, the patient was discharged the $7^{\text {th }}$ day postoperatively.

\section{DISCUSSIONS}

The vestibular syndrome in this particular case was due to a floating and extruded electrode array, although the cochleostomy was correctly performed and was not too large (0.8 $\mathrm{mm})$, the FLEX28 and Compressed arrays differ very slightly in diameter ( 0.7 vs $0.8 \mathrm{~mm}$ ), and the sealing of the cochleostomy with muscle tissue was satisfactory. We consider the appareance of this phenomenon in direct connection to the quality of the inserted electrode (probably too elastic and smooth, with conical shape, having an increased tendance towards migration out of the cochlea). Fixation of the electrode to a stable osseous margin is a solution that we have been using since this episode with this type of electrode. Other techniques of stabilising the implant and the electrode array have been cited into the literature $(5,6)$ : bony hook at the mastoidectomy edge, bone pate harvested during surgery and obliterating the mastoid external opening.

The electrode array can also be extruded in cases of cochlear implant revision surgeries where device body needs to be fixed or migrated (7). A thorough check of the electrode position becomes mandatory in these cases, before ending the procedure.

\section{CONCLUSIONS}

Different types of cochlear implants pose different surgical problems when it comes to stability and reliability of the electrode. Although the final rehabilitation results of the patients seem similar in many ways, preventing postoperative complications like this one presented in this paper is obtained by understanding the properties of the devices used and adapting them to each patient's anatomy.

Conflict of interest: none declared Financial support: none declared 


\section{REFERENCES}

1. Fina M. et al. Vestibular dysfunction after cochlear implantation. Otol Neurotol, 2003. 24(2): p. 234-42; discussion 242.

2. Au G., Gibson W. Cochlear implantation in children with large vestibular aqueduct syndrome. Am J Otol, 1999. 20(2): p. 183-6.

3. Enticott J.C. et al. Cochlear implant and vestibular function. Otol Neurotol, 2006. 27(6): p. 824-30.
4. Bonucci A.S. et al. Vestibular function in cochlear implant users. Braz $J$ Otorhinolaryngol, 2008. 74(2): p. 273-8.

5. Roberson J.B. et al. Modifications of standard cochlear implantation techniques for children under 18 months of age. Cochlear Implants Int, 2006. 7(4): p. 207-13.

6. Fedoseev V.I. (Comparative analysis of approaches to surgical cochlear implantation in adults and children). Vestn Otorinolaringol, 2003(6): p. 35-7.

7. Gheorghe D.C., Zamfir-Chiru-Anton A. Complications in cochlear implant surgery. J Med Life, 2015. 8(3): p. 329-32. 